\title{
Methods matter: The relationship between strength and hypertrophy depends on methods of measurement and analysis
}

\author{
Andrew D Vigotsky ${ }^{\text {Corresp., }}{ }^{1}$, Brad J Schoenfeld ${ }^{2}$, Christian Than ${ }^{3}$, J Mark Brown ${ }^{3}$ \\ 1 Department of Biomedical Engineering, Northwestern University, Evanston, Illinois, United States of America \\ 3 School of Biomedical Sciences, University of Queensland, St. Lucia, Queensland, Australia \\ Corresponding Author: Andrew D Vigotsky \\ Email address: avigotsky@gmail.com
}

Purpose: The relationship between changes in muscle size and strength may be affected by both measurement and statistical approaches, but their effects have not been fully considered or quantified. Therefore, the purpose of this investigation was to explore how different methods of measurement and analysis can affect inferences surrounding the relationship between hypertrophy and strength gain.

Methods: Data from a previous study - in which participants performed eight weeks of elbow flexor training, followed by an 8-week period of detraining- were reanalyzed using different statistical models, including standard between-subject correlations, analysis of covariance, and hierarchical linear modeling.

Results: The associative relationship between strength and hypertrophy is highly dependent upon both method/site of measurement and analysis; large differences in variance accounted for (VAF) by the statistical models were observed (VAF $=0-24.1 \%$ ). Different sites and measurements of muscle size showed a range of correlations coefficients with one another $(r=0.326-0.945)$. Finally, exploratory analyses revealed moderate-to-strong relationships between within-individual strength-hypertrophy relationships and strength gained over the training period $(\rho=0.36-0.55)$.

Conclusions: Methods of measurement and analysis greatly influence the conclusions that may be drawn from a given dataset. Analyses that do not account for inter-individual differences may underestimate the relationship between hypertrophy and strength gain, and different methods of assessing muscle size will produce different results. It is suggested that robust experimental designs and analysis techniques, which control for different mechanistic sources of strength gain and inter-individual differences (e.g., muscle moment arms, muscle architecture, activation, and normalized muscle force), be employed in future investigations. 
1 Methods Matter: The relationship between strength and hypertrophy depends on methods

2 of measurement and analysis

3

4 Andrew D. Vigotsky ${ }^{1}$, Brad J. Schoenfeld ${ }^{2}$, Christian Than ${ }^{3}$, J. Mark Brown ${ }^{3}$

5

$6 \quad{ }^{1}$ Department of Biomedical Engineering, Northwestern University, Evanston, IL, USA

$7 \quad{ }^{2}$ Department of Health Science, Lehman College, Bronx, NY, USA

$8 \quad{ }^{3}$ School of Biomedical Sciences, The University of Queensland, St. Lucia 4072, Australia

18 Corresponding author:

19 Andrew Vigotsky

20 ORCID: 0000-0003-3166-0688

21 avigotsky@gmail.com

22

23 Running title: Methods Matter 


\section{ABSTRACT}

26 Purpose: The relationship between changes in muscle size and strength may be affected by both measurement and statistical approaches, but their effects have not been fully considered or quantified. Therefore, the purpose of this investigation was to explore how different methods of measurement and analysis can affect inferences surrounding the relationship between hypertrophy and strength gain.

31 Methods: Data from a previous study - in which participants performed eight weeks of elbow 32 flexor training, followed by an 8-week period of detraining - were reanalyzed using different 33 statistical models, including standard between-subject correlations, analysis of covariance, and 34 hierarchical linear modeling.

35 Results: The associative relationship between strength and hypertrophy is highly dependent upon both method/site of measurement and analysis; large differences in variance accounted for (VAF) 37 by the statistical models were observed (VAF $=0-24.1 \%)$. Different sites and measurements of 38 muscle size showed a range of correlations coefficients with one another $(r=0.326-0.945)$. 39 Finally, exploratory analyses revealed moderate-to-strong relationships between within-individual strength-hypertrophy relationships and strength gained over the training period $(\rho=0.36-0.55)$.

41 Conclusions: Methods of measurement and analysis greatly influence the conclusions that may be 42 drawn from a given dataset. Analyses that do not account for inter-individual differences may 43 underestimate the relationship between hypertrophy and strength gain, and different methods of 44 assessing muscle size will produce different results. It is suggested that robust experimental 45 designs and analysis techniques, which control for different mechanistic sources of strength gain 46 and inter-individual differences (e.g., muscle moment arms, muscle architecture, activation, and normalized muscle force), be employed in future investigations. 
50 Introduction

51

52

53

54

The combined actions of neural input, muscles, and the joint(s) about which those muscles act serve to produce sufficient endpoint force for physical function, allowing the performance of activities of daily living, as well as the spectrum of athletic endeavors. Due to the complexity of the neuromuscular and musculoskeletal systems, many factors can influence strength, including, but not limited to, muscle moment arm, muscle size, activation, muscle architecture, and normalized muscle force (or specific tension) (Vigotsky et al. 2015). Muscle size is of particular interest, as 1) it is highly plastic (Fluck \& Hoppeler 2003) and 2) a clear positive relationship exists between baseline muscle cross-sectional area (CSA) and strength, with greater CSAs correlating with greater strength capacities (Maughan \& Nimmo 1984; Maughan et al. 1984; Schantz et al. 1983). However, this relationship is not necessarily linear, as several additional factors interactively influence strength capacity (Vigotsky et al. 2015); studying the role of and relationship between muscle size and strength is therefore less straightforward under longitudinal contexts.

While the cross-sectional correlation between muscle mass and strength remains wellestablished, some researchers have recently challenged the belief that resistance training (RT) induced hypertrophy significantly impacts the ability to produce force, claiming improvements in these outcomes are separate and unrelated adaptations (Buckner et al. 2016a). Indeed, data remain somewhat equivocal on the relationship between changes in size and changes in strength resulting from regimented RT: A considerable range of correlation coefficients have been observed, from $\sim 0$ to $\sim 0.6$ (Ahtiainen et al. 2016; Appleby et al. 2012; Baker et al. 1994; Balshaw et al. 2017; Cribb et al. 2007; Erskine et al. 2014; Erskine et al. 2010; Loenneke et al. 2017; Maeo et al. 2018; Pope et al. 2016; Rasch \& Morehouse 1957; Watanabe et al. 2018). The discrepancies in findings between studies may be related, in part, to the statistical measures employed to analyze relationships between muscle hypertrophy and strength gain. For instance, analyses in a majority of studies are based on between-subject data using only two time points, but within-subject analyses are more appropriate for the question at hand. Inferentially, drawing individual-level conclusions from group-level data is a statistical fallacy, known as the ecological fallacy (Robinson 1950). Pragmatically, this problem can be better understood by differentiating between the question that each analysis addresses. Between-subject analyses answer the question, "Do those who grow more also get stronger than those who grow less?" Conversely, within-subject 
81 analyses answer the question, "Is the growth of one's muscle related to their increases in strength?"

82 Due to individual differences, the former (between-subject) may not necessarily map to the latter 83 (within-subject). For example, if subject A has a 30\% larger muscle moment arm than subject B, 84 then one may expect subject A to have a 30\% greater slope between increases in muscular strength 85 (force) and externally-measured strength (moment), all else being equal. To address the ecological 86 fallacy and answer the within-subject question, more sophisticated statistical approaches are needed (Goldstein 2011; Jackson et al. 2006; Robinson 1950).

A hierarchical approach can assist in avoiding the pitfall of the ecological fallacy 89 (Goldstein 2011; Jackson et al. 2006). Traditionally, each participant's change in strength and change in size, from pre- to post-intervention, are calculated and regressed among one another (Ahtiainen et al. 2016; Appleby et al. 2012; Baker et al. 1994; Balshaw et al. 2017; Cribb et al. 2007; Erskine et al. 2014; Erskine et al. 2010; Maeo et al. 2018; Pope et al. 2016; Rasch \& Morehouse 1957; Watanabe et al. 2018). However, a hierarchical modeling approach allows for one to look at time points nested within participants, such that each participant's points are kept "separate" from other participants (Gelman \& Hill 2007; Goldstein 2011; Raudenbush \& Bryk 2002). Within the hierarchical model, each participant can receive varying intercepts and/or varying slopes, which allows for inter-individual differences to be appropriately accounted for (Gelman \& Hill 2007; Goldstein 2011; Raudenbush \& Bryk 2002). To carry out hierarchical modeling with varying slopes and intercepts, multiple $(\geq 3)$ time points are required (i.e., to quantify model variance), so most training datasets cannot be used to answer this question, as a majority only collect data at two time points (pre- and post-intervention). To date, only one study has employed a within-subject analysis: Loenneke et al. (2017) used analysis of covariance (ANCOVA) (Bland \& Altman 1995a) and found appreciably greater coefficients of determination in within- relative to between-subject models for the same muscle and strength test $\left(e . g ., \mathrm{R}^{2}=\right.$ 0.004 vs. 0.35). However, in contrast to hierarchical linear models, ANCOVA has an affine assumption; participants receive different intercepts, but all are constrained to the same slope (Bland \& Altman 1995a). Therefore, further work is needed to understand how model choice affects the strength of the relationship between hypertrophy and changes in strength.

The purpose of this study was to investigate the relationship between changes in muscle size and strength in the elbow flexors using a variety of statistical and measurement approaches,

111 while also employing both between- and within-subject analyses over multiple time-points during 
112 periods of both training and detraining. It was hypothesized that different statistical models would

113 produce different outcomes, with between-subject correlations showing the weakest relationships

114 and hierarchical linear modeling showing the strongest.

115

116 Methods

\section{Participants}

118 The study reanalyzed data from a previously published study, the methods of which have 119 been described (Than et al. 2016). In brief, young, recreationally active individuals (mean $\pm \mathrm{SD}$, 120 age $=24 \pm 3$ years, BMI $=22 \pm 2, n=19$ ) were recruited for participation in the study. Participants 121 reported exercising at least 3 times per week via various sporting activities but did not perform 122 resistance training for the elbow flexors. Informed consent was obtained for all participants. The 123 original study was approved by the University of Queensland Medical Research Ethics Committee 124 (no. 2014001416).

125

126 Muscle size

127 Measures of muscle thickness were obtained via B-mode ultrasound imaging (Mindray 128 DP-50) using a $7.5 \mathrm{MHz}$ linear transducer probe. Images were taken at baseline and after each 129 week of training throughout the 16-week study period. Scanning was carried out by a trained 130 sonographer on both the dominant and non-dominant elbow flexors at 30,50 , and $70 \%$ of total 131 length of the biceps brachii whilst participants were seated with the antebrachium in a neutral 132 position. After weeks 4,8 , and 16, CSA scans were acquired for both upper limbs via panoramic 133 B-mode ultrasound (S3000 Siemens/Acuson system) using a 4-9 MHz linear transducer operating 134 at $9 \mathrm{MHz}$. Imaging for CSA was obtained via lateral acquisition at 50\% width of the biceps brachii. 135 Values for both muscle thickness and CSA were determined using ImageJ (version 1.48; National 136 Institutes of Health, Bethesda, MD). Muscle thickness was not assessed for Week 4 due to a 137 conflict in scheduling with CSA ultrasounds. All ultrasound measures were completed by a paid 138 qualified professional, and not by the researchers of the paper. If the probe lost contact at any point 139 during the measurement, the measurement was retaken. Test-retest intraclass correlation 140 coefficients (ICC; model 2,1) of 0.99 and 0.97 for CSA and muscle thickness, respectively, have 141 been previously reported (Jenkins et al. 2015). Because an $\operatorname{ICC}(2,1)$ model was used, these results 
142 are generalizable to the experienced rater in this study (Koo \& Li 2016).

\section{Resistance training protocol}

145 Resistance training for the non-dominant brachium was carried out five days per week for

146 the initial 8 weeks of the study, followed by a subsequent 8 -week detraining period. Training

147 consisted of unilateral dumbbell elbow flexion performed with a supinated forearm. During each

148 session, participants performed 9 sets of 12 repetitions with a 90-second rest interval afforded

149 between sets. Loads were based on maximal voluntary isometric contraction (MVIC) values that

150 were obtained each week using a Sundoo SN Analogue Force Gauge (model number SN-500) at

$15190^{\circ}$ elbow flexion. Subjects began each workout using $70 \%$ of that week's MVIC recording. If the

152 full number of target repetitions (i.e., 12) was not achieved on a given set, the load was lowered to

153 the next level of load until completion - e.g., if a participant achieved 8 repetitions at $70 \%$, the

154 load was decreased to $50 \%$ so that all 12 reps could be performed. Loads were progressively

155 lowered on successive sets to $50 \%$ and $30 \%$ of MVIC as needed so that subjects could complete

156 the target repetition range with proper form. The dominant brachium of each subject served as the

157 control for the study throughout the training and detraining periods. Subjects were instructed to

158 refrain from exercise involving the elbow flexors, other than activities of daily living, throughout

159 the 16-week study period.

160

161

\section{Statistical analysis}

162

Several statistical analyses were carried out to investigate how methods of both measurement and analysis may affect the conclusions drawn from a study investigating the relationship between strength and hypertrophy. All analyses were carried out in R (version 3.4.3) (R Core Development Team 2017). First, standard bivariate linear regression analyses of pre- and post-measures were utilized to investigate the relationship between muscle size (thickness or CSA) and strength, using a between-subject model. This was done for two different conditions: training and detraining. For each condition, a data point ( $\left.\Delta_{\text {size }}, \Delta_{\text {strength }}\right)$ was calculated for each participant, where, in the general case, $\Delta=$ post - pre, where pre and post are the values before and after a given condition (training or detraining), respectively, as has been done in a number of previous

171 investigations (Ahtiainen et al. 2016; Erskine et al. 2014; Loenneke et al. 2017). Second, an

172 ANCOVA was utilized to replicate the method of analysis used by Loenneke et al. (2017). In this 
173 analysis, strength was treated as a dependent variable, participants were treated as a categorical

174 factor (dummy-coded), and size was treated as a covariate. Variance accounted for (VAF) was

175 calculated using the formula $\mathrm{VAF}=\frac{\mathrm{SS}_{\text {size }}}{\mathrm{SS}_{\text {size }}+\mathrm{SS}_{\text {residual }}}$, where $\mathrm{SS}$ is type III sum of squares (Bland \&

176 Altman 1995a). This is equivalent to a partial $\eta^{2}$ for the size covariate. Lastly, because the

177 ANCOVA method has a number of assumptions and does not allow for varying slopes, a more

178 robust hierarchical linear model was used for the final analysis (Quené \& van den Bergh 2004). In

179 this analysis, the outcome measure $\left(y_{i j}\right)$ was the net joint moment during MVIC, and muscle size

180 was used as a level-one predictor variable $\left(x_{i j}\right)$, which were group-mean centered for analyses.

181 Subject was treated as a level-two variable. Finally, varied slopes and intercepts were permitted,

182 creating the final model:

183

184

Level 1

185

$$
\mathrm{y}_{\mathrm{ij}}=\beta_{0 \mathrm{j}}+\beta_{1 \mathrm{j}} \mathrm{x}_{\mathrm{ij}}+\epsilon_{\mathrm{ij}}
$$

186

188

\section{Level 2}

$$
\beta_{0 \mathrm{j}}=\gamma_{00}+\mathrm{r}_{0 \mathrm{j}}
$$

$$
\beta_{1 \mathrm{j}}=\gamma_{10}+\mathrm{r}_{1 \mathrm{j}}
$$

189

190 The model was fit using restricted maximum likelihood in the lme4 package (Bates et al. 2015).

191 Sample variance of the residuals $\left(s^{2}\right)$ were used to calculate VAF (or $\mathrm{R}^{2}$ ) using the following

192 formula: $\mathrm{VAF}=1-\frac{\mathrm{s}^{2}}{\mathrm{~s}_{\text {uncond }}^{2}}$, where $\frac{\mathrm{s}_{\text {uncond }}}{2}$ is the sample variance of the residuals in the unconditional

193 model, which contained only varied intercepts and no fixed effects (i.e., the same model, but with

$\left.194 \beta_{1 j}=0\right)$. This approach is mathematically equivalent to the VAF found for the ANCOVA using

195 type III sums of squares (see Appendix A). Intraclass correlation coefficients (ICC) were

196 calculated on the unconditional models to estimate the proportion of original variance explained

197 by subject. To estimate 95\% confidence intervals (CI) of the VAFs, each model was bootstrapped

1982000 times with replacement. The 0.025 and 0.975 quantiles of the VAF estimates were calculated

199 as the lower and upper bounds of each estimate's 95\% CI.

200 To understand how the different measures of hypertrophy relate to one another, within-

201 and between-subject correlation matrices were constructed using the different thickness measures 
202 and CSA. The between-subject analysis included all thickness and CSA measures, across all 203 subjects, for any time point at which both CSA and thickness were measured. The within-subject 204 correlation matrix was constructed in a similar manner: 1) a correlation coefficient was calculated 205 for each participant $\left.\left(r_{i}\right) ; 2\right)$ using a Fisher $z$-transformation, $r_{i}$ was transformed to a $z$-score $\left.\left(z_{i}\right) ; 3\right)$ 206 a weighted average was obtained using the number of points $\left(n_{i}\right)$ from each participant $\left(\overline{\mathrm{z}}=\frac{\sum \mathrm{z}_{\mathrm{i}}\left(\mathrm{n}_{\mathrm{i}}-3\right)}{\sum\left(\mathrm{n}_{\mathrm{i}}-3\right)}\right.$, 207 for $i$ participants); and 4) $\bar{z}$ was transformed back to Pearson's $r$ (Borenstein et al. 2009; Cooper et 208 al. 2009; Corey et al. 1998; Hedges \& Olkin 1985). Because CSA measures were only taken with thickness at two time points, within-subject correlation coefficients could not be estimated between CSA and muscle thickness.

211 Further exploratory analyses were performed to investigate if those with stronger strength212 hypertrophy relationships also got stronger. To do this, Pearson correlation coefficients were 213 calculated for each individual across the entire study (i.e., including both training and detraining 214 periods). The resulting correlation coefficients were then correlated with $\Delta_{\text {strength }}$ from the training 215 period using Spearman's rank-order correlations $(\rho)$. Spearman's $\rho$ was used due to the 216 heteroscedastic nature of the residuals. Qualitative interpretations of correlation coefficients and 217 VAFs can be found in Table 1, which are in accordance with Hopkins (2002). R code for all 218 procedures can be found in the Supplemental Files.

219

* Table 1 here *

221

222

\section{Results}

224 Differences in VAFs ranged from zero to an order of magnitude (Table 2). Similar differences 225 were also observed between different statistical models for a given measure (Table 2). Intraclass correlation coefficients from the hierarchical linear models suggest that most of the original variance could be accounted for by including a level for subject $(\mathrm{ICC}=0.89-0.91)$. Heterogeneity in correlation coefficients was observed when comparing different measures of muscle thickness, which ranged from $r=0.503$ to $r=0.945$ for between-subject correlations and from $r=0.326$ to $r=0.875$ for weighted within-subject correlations (Table 3). Finally, Pearson's $r$ of each

231 individual's strength-hypertrophy relationship was a moderate to strong predictor of strength for 
232 all measurements $\left(\mathrm{US}_{30 \%} \rho=0.644 ; \mathrm{US}_{50 \%} \rho=0.356 ; \mathrm{US}_{70 \%} \rho=0.413 ; \mathrm{US}_{\text {avg }} \rho=0.480 ; \operatorname{CSA} \rho=\right.$ $2330.449)$.

234

$235 *$ Table 2 here *

236

$237 *$ Table 3 here *

238

239

\section{Discussion}

To the authors' knowledge, this is the first study to investigate the relationship between hypertrophy and changes in muscle strength using hierarchical linear modeling, which allows for robust within-individual analysis, in addition to the use of multiple types of measures of muscle size. Our results demonstrate that not only does measurement approach substantially affect outcomes, but so does the type of statistical model employed. These findings have important methodological implications for improving our understanding of the associative relationship between hypertrophy and changes in strength.

Previous literature has approached the question of how changes in muscle size relate to changes in strength from a between-subject perspective. However, it can be argued that a repeatedmeasures design allows for a more direct evaluation of the strength-hypertrophy relationship. Individual differences in muscle moment arms (MA), normalized muscle force (NMF), pennation angles $\left(\theta_{\mathrm{p}}\right)$, voluntary activation $(\alpha)$, et cetera will greatly confound the relative relationship between changes in strength and muscle size (in this case, physiological CSA(PCSA)). All of the aforementioned components are multipliers in the formula used to calculate a muscle's contribution to a joint moment $\left(\mathrm{M}=\alpha \cdot \mathrm{PCSA} \cdot \mathrm{NMF} \cdot \cos \theta_{\mathrm{p}} \cdot \mathrm{MA}\right)$ (Vigotsky et al. 2015). To date, only one previous investigation has utilized a quantitative within-subject approach to investigate the relationship between hypertrophy and changes in strength (Loenneke et al. 2017); although, qualitative within-subject changes are depicted in a classic study by DeLorme (1945). Specifically, Loenneke et al. (2017) employed an ANCOVA with subject as a factor and muscle size as a covariate; from the resulting sum of squares, VAF could be calculated (Bland \& Altman 1995a). ANCOVA is limited, however, in that it, in its basic form, assumes parallelism between all relationships, has several assumptions that may confound results (e.g., sphericity, compound symmetry, and homoscedasticity), and is not robust to missing data points (Bland \& 
263 Altman 1995a; Bland \& Altman 1995b; Quené \& van den Bergh 2004). The parallel or affine 264 assumption is of particular interest because there are several heterogeneities that confound this 265 assumption (i.e., $\alpha, \mathrm{MA}, \mathrm{NMF}$, and $\theta_{\mathrm{p}}$ ). Repeated-measures hierarchical models are a robust way 266 to investigate longitudinal relationships within a group or person (Gelman \& Hill 2007; 267 Raudenbush \& Bryk 2002). By comparing these statistical models, a clear difference is apparent 268 (Table 2). For all measurements, the hierarchical linear model resulted in greater VAFs than the 269 ANCOVA (Table 2). These differences may be due to the hierarchical linear model allowing for 270 varying slopes or, alternatively, some of the inherent assumptions and limitations of ANCOVAs 271 (Quené \& van den Bergh 2004). Interestingly, the VAFs found in this present study are much 272 lower than those found by Loenneke et al. (2017). It is unclear from where these differences arise; 273 that is, if they are due to measurement technique, differences in mechanisms of strength gain, 274 differences in upper vs. lower extremities, or some other factor. However, our data provide a 275 methodological proof of principle by delineating how different statistical models may drastically 276 affect the conclusions formed from a given dataset, even when performed on the same set of regressors. Due to the robustness of hierarchical linear models, it is recommended that such analyses are used over ANCOVAs for future investigations with similar methods.

How muscle size is assessed will likely affect the strength of the relationship between 280 changes in muscle size and strength. The measurement techniques utilized by previous and present investigations (Ahtiainen et al. 2016; Appleby et al. 2012; Baker et al. 1994; Balshaw et al. 2017; Buckner et al. 2016a; Cribb et al. 2007; Erskine et al. 2014; Loenneke et al. 2017; Pope et al. 2016) have been limited in that they do not account for changes in architectural characteristics (Lieber \& Ward 2011). There are several ways to measure muscle size, including limb circumference (DeLorme 1945), estimates of total and segmental muscle mass (dual-energy X-ray absorptiometry and bioelectrical impedance analysis) (Karelis et al. 2013), muscle thickness (Than et al. 2016), anatomical CSA (Erskine et al. 2014; Trezise et al. 2016), muscle volume (Balshaw et al. 2017; Erskine et al. 2014; Erskine et al. 2010), and PCSA (Erskine et al. 2010). There are strong physiological and mechanical rationales with basic science evidence to suggest that not all of these measures are equal, even when accounting for measurement error (Lieber \& Ward 2011; Powell et al. 1984). For example, although muscle volume appears to be a strong predictor of strength in some contexts (even greater than anatomical CSA) (Akagi et al. 2009; Fukunaga et al. 2001), it 
294 intra-muscular variation in architecture (Blazevich et al. 2006; Lieber \& Ward 2011; Ward et al. 295 2009) and adaptation (Earp et al. 2015; Ema et al. 2013; Franchi et al. 2017; Narici et al. 1996; 296 Wakahara et al. 2013; Wakahara et al. 2012). Muscle volume is not only sensitive to changes in 297 sarcomeres in parallel (PCSA), but also sarcomeres in series (fiber length). Sarcomeres in parallel 298 will contribute to the magnitude of force production, while sarcomeres in series will affect the 299 shapes of the force-length and force-velocity curves. Functionally speaking, not all muscle volume 300 is equal (Lieber \& Ward 2011). Importantly, in series hypertrophy appears to be limited to the 301 initial weeks of commencing resistance training, further reinforcing potential issues when 302 extrapolating correlative findings from novice to trained individuals (Blazevich et al. 2007). 303 Similarly, thickness and anatomical CSA, as measured in this study, are also limited, as they only 304 represent one part of the muscle and do not account for the intricacies of muscle architecture. This 305 is further evidenced by Franchi et al. (2017), who found that, cross-sectionally, muscle thickness, 306 anatomical CSA, and muscle volume are related, but the relative changes between muscle 307 thickness and muscle volume did not strongly correlate following a training period. This is 308 important when considering the formula for PCSA, in that the volume of the entire muscle must 309 be taken into account (Lieber \& Ward 2011); not just thickness or anatomical CSA. Moreover, the 310 variability in correlation coefficients between these measures may be a cause for concern (Table 3113 ), in that it suggests not all measures of muscle size are necessarily capturing the same effects, 312 which is elucidated further by the statistical models (Table 2). Since PCSA has been shown to be 313 a strong predictor of force production both in vivo (Fukunaga et al. 1996) and in vitro (Powell et 314 al. 1984), it is considered the gold standard for relating muscle form (architecture) to function 315 (force production) (Lieber \& Ward 2011). PCSA is, in essence, the "effective" CSA, as it is the 316 average CSA perpendicular to the fibers' line of action. Thus, PCSA controls for pennation and is 317 representative of the number of sarcomeres in parallel, making it highly indicative of a muscle's 318 potential to generate force through the tendon (Lieber \& Ward 2011). It is imperative to consider 319 these differences in measurement techniques in the context of this study and similar investigations 320 (Ahtiainen et al. 2016; Erskine et al. 2014; Erskine et al. 2010; Loenneke et al. 2017). Although 321 this study (Table 2) and others (Loenneke et al. 2017) have observed what is analogous to a strong 322 correlation $(r \geq 0.5)$ (Hopkins 2002) with repeated-measures designs, substandard measurements 323 of muscle size were used in the present study. Therefore, it is likely that PCSA measurements 324 would produce different results (Aagaard et al. 2001). While PCSA is expensive to obtain and 
325 typically relies on MRI, newer technologies, such as 3D ultrasound, show promise as valid, 326 affordable alternatives to MRI for estimating muscle volume and PCSA (Barber et al. 2009; Barber 327 et al. 2011; Haberfehlner et al. 2016). Moving forward, it seems prudent that investigators utilize 328 PCSA rather than other measures of muscle size, as the theory that hypertrophy leads to strength 329 gains is predicated on this measure rather than other measures of muscle size.

330 The question of how changes in strength and changes in muscle size are related is one with broad clinical implications, ranging from the treatment and prevention of sarcopenia and dynapenia to exercise prescription for strength athletes. Clinically, if changes in muscle size are not important

333 for strength, then exercise programs need not focus on variables that are more important for 334 hypertrophy than strength, such as volume (Ralston et al. 2017; Schoenfeld et al. 2017). Changes in strength do indeed arise from non-hypertrophic factors (Folland \& Williams 2007), including a myriad of neural adaptations (Enoka 1988), in addition to changes in muscle moment arms

337 (Sugisaki et al. 2015; Vigotsky et al. 2015) and normalized muscle force production (Erskine et al. 2010), in which lateral force transmission has been suggested to play a role (Jones et al. 1989). This implies that changes in strength are interactive rather than linear. As such, how this relationship is investigated and modeled should reflect such complexities. First, with more reductionist strength testing (i.e., single-joint isometric testing), it can be argued that the "skill" component of strength is less relevant (as opposed to one-repetition maximum tests (Buckner et al. 2016b)), since little coordination is necessary and even untrained individuals see little-to-no changes in voluntary activation and co-contraction (Behm 1995; Erskine et al. 2014; Erskine et al. 2010; Noorkoiv et al. 2014). Moreover, neural measures, such as voluntary activation, can be more accurately assessed during isometric efforts than during dynamic efforts (Farina 2006; Vigotsky et al. 2017) and thus can more easily be incorporated into a final model. Second, measures of muscle size should reflect those in the model (i.e., using PCSA). While this is expensive and time consuming, it will provide more appropriate biomechanical insight (Lieber \& Ward 2011). Third, moment arm measures should be subject-specific and occur over the duration of an experiment, as moment arms may change with training (Sugisaki et al. 2015; Vigotsky et al. 2015). Finally, longer duration studies may be more appropriate for several reasons: 1) individual response trajectories will vary, as evidenced by the high ICCs in this present investigation and the heterogeneous rank orders between time points in previous work (Churchward-Venne et al. 2015); 2) edema can greatly confound gross imaging measures of muscle size, depending on when the measurements 
356 are performed (Damas et al. 2016); 3) the magnitude of the difference between measurement points

357 will be greater, which in turn will decrease the relative role of measurement error in parameter and

358 VAF estimates (Fuller 1987); and 4) to understand the extent to which contributions may or may

359 not change over time. While this present study did not incorporate these recommendations, since

360 it was based on previously collected data (Than et al. 2016), future studies should do so to properly

361 isolate the associative contribution of muscle size (PCSA) to strength increases.

362 Thus far, our discussion has primarily focused on the associative, rather than causal,

363 relationship between hypertrophy and strength gain. A conducive discussion of the causal nature

364 of this relationship requires an operational definition of causality. In formal logic, causality is often

365 broken down into two conditions: 1) necessary conditions, which state that $B$ will not occur without

$366 A$ ("if not $A$, then not $B$ "); and 2) sufficient conditions, which state that $A$ will result in $B$ ("if $A$,

367 then $B$ ") (Epp 2011; Hall 1987). However, a less formal concept of causality is also possible

368 without these conditions having been met, in the form of contributory causality. A contributory

369 cause is neither necessary nor sufficient (Hall 1987; Riegelman 1979). Those who experience an

370 effect need not experience its putative cause, and those who experience the putative cause need

371 not experience its effect (Riegelman 1979). For instance, although smoking causes lung cancer,

372 not all of those who smoke develop lung cancer (i.e., it is not sufficient), and not all of those who

373 develop lung cancer are smokers (i.e., it is not necessary); therefore, smoking may be viewed as a

374 contributory cause of lung cancer (Riegelman 1979). The arguments put forth by Buckner et al.

375 (2016a); Dankel et al. (2018); Mattocks et al. (2017) do indeed rule out hypertrophy as being a

376 necessary or sufficient cause for strength gain, but we suggest that the contributory nature of

377 hypertrophy to strength should not be dismissed on this basis. In other words, changes in strength

378 can occur without changes in muscle size and vice versa, but this does not preclude muscle size

379 from contributing to strength. Experimentally, it is important to consider the emergent, nonlinear,

380 and interactive properties of strength; there are many moving parts that should be accounted for

381 when attempting to understand such a complex system, which may concurrently change in

382 different directions (e.g., increase in size but decrease agonist activation). Indeed, a systems rather

383 than reductionist approach may be most appropriate for understanding strength emergence. In

384 studying this system, it is necessary to measure all factors (confounders) that may contribute to

385 strength to truly understand the role of hypertrophy, especially because different protocols may

386 elicit differential adaptations (Jenkins et al. 2017). Thus, longitudinal, within-subject studies that 
387 incorporate all of the measures included in the formula to determine strength (PCSA, MA, 388 activation and co-contraction, synergist characteristics, and NMF) are likely needed to better 389 understand the emergent properties of strength. Finally, because the problem is so complex, the 390 contributory role of hypertrophy in strength gain may not be able to be fully established from one 391 study or line of evidence. Instead, a body of literature consisting of many forms of evidence392 ranging from animal and agent-based models to observational and experimental human studies393 may be required to elucidate the contributory role of hypertrophy in strength gain.

This study and its discussion have focused primarily on single muscle group hypertrophy and single-joint isometric strength gain. The larger question of multi-joint and dynamic strength gain is perhaps more relevant, but unfortunately much more complex (Vigotsky et al. 2018). Starting with relatively simpler systems and research questions may bear more fruit, while also providing a conceptual basis that can be used when studying more complex systems and research questions.

This is the first study to utilize repeated-measures hierarchical linear modeling to investigate the relationship between muscle size and strength. We herein demonstrate that repeated-measures hierarchical linear models produce different results than other within-subject models (ANCOVA), in addition to between-subject models, which is in line with previous work by Loenneke et al. (2017). Moreover, it was found that different measures of muscle size can produce vastly different results. As such, we have advocated for more rigorous and reductionist experimental designs to better understand the mechanistic origins of single-joint strength following exercise programs, by suggesting that researchers measure PCSA and single-joint isometric strength, in addition to potential confounding variables ${ }^{1}$. These findings are important for the interpretation of previous studies, in addition to the design of future studies, on this same topic.

\section{Conclusions}

412 The strength of the associational relationship between muscle hypertrophy and strength gain is 413 highly dependent upon the statistical model employed. We have demonstrated that hierarchical 414 linear modeling, which allows for varying slopes and intercepts, provides greater estimates of the 415 strength of the relationship between muscle hypertrophy and strength gain. Moreover, different

\footnotetext{
${ }^{1}$ Note that these recommendations only apply to studies that are investigating the strength-hypertrophy relationship with a reductionist approach. We are in no way suggesting that PCSA and single-joint isometric measures be used for all resistance training studies.
} 
416 assessments of muscle size do not perfectly correlate, and therefore, different methods of 417 assessment may lead to different conclusions. These findings should be taken into consideration 418 when planning and interpreting studies on the relationship between muscle hypertrophy and 419 strength gain. 
421

422

423

424

425

426

427

428

429

430

431

432

433

434

435

436

437

438

439

440

441

442

443

444

445

446

447

448

449

450

451

452

453

454

455

456

457

458

459

460

461

462

\section{References}

Aagaard P, Andersen JL, Dyhre-Poulsen P, Leffers AM, Wagner A, Magnusson SP, HalkjaerKristensen J, and Simonsen EB. 2001. A mechanism for increased contractile strength of human pennate muscle in response to strength training: changes in muscle architecture. The Journal of physiology 534:613-623. 10.1111/j.1469-7793.2001.t01-1-00613.x

Ahtiainen JP, Walker S, Peltonen H, Holviala J, Sillanpaa E, Karavirta L, Sallinen J, Mikkola J, Valkeinen H, Mero A, Hulmi JJ, and Hakkinen K. 2016. Heterogeneity in resistance traininginduced muscle strength and mass responses in men and women of different ages. Age (Dordr) 38:10. 10.1007/s11357-015-9870-1

Akagi R, Takai Y, Ohta M, Kanehisa H, Kawakami Y, and Fukunaga T. 2009. Muscle volume compared to cross-sectional area is more appropriate for evaluating muscle strength in young and elderly individuals. Age and Ageing 38:564-569. 10.1093/ageing/afp122

Appleby B, Newton RU, and Cormie P. 2012. Changes in strength over a 2-year period in professional rugby union players. Journal of Strength and Conditioning Research 26:25382546. 10.1519/JSC.0b013e31823f8b86

Baker D, Wilson G, and Carlyon R. 1994. Periodization: The Effect on Strength of Manipulating Volume and Intensity. Journal of Strength and Conditioning Research 8:235-242.

Balshaw TG, Massey GJ, Maden-Wilkinson TM, Morales-Artacho AJ, McKeown A, Appleby CL, and Folland JP. 2017. Changes in agonist neural drive, hypertrophy and pre-training strength all contribute to the individual strength gains after resistance training. European Journal of Applied Physiology 117:631-640. 10.1007/s00421-017-3560-x

Barber L, Barrett R, and Lichtwark G. 2009. Validation of a freehand 3D ultrasound system for morphological measures of the medial gastrocnemius muscle. Journal of Biomechanics 42:1313-1319. 10.1016/j.jbiomech.2009.03.005

Barber L, Hastings-Ison T, Baker R, Barrett R, and Lichtwark G. 2011. Medial gastrocnemius muscle volume and fascicle length in children aged 2 to 5 years with cerebral palsy. Developmental Medicine and Child Neurology 53:543-548. 10.1111/j.14698749.2011.03913.x

Bates D, Mächler M, Bolker B, and Walker S. 2015. Fitting Linear Mixed-Effects Models Using Ime4. 2015 67:48. 10.18637/jss.v067.i01

Baxter JR, and Piazza SJ. 2014. Plantar flexor moment arm and muscle volume predict torquegenerating capacity in young men. Journal of Applied Physiology 116:538-544. 10.1152/japplphysiol.01140.2013

Behm DG. 1995. Neuromuscular implications and applications of resistance training. Journal of Strength and Conditioning Research 9:264-274. 10.1097/00124278-199511000-00014

Bland JM, and Altman DG. 1995a. Calculating correlation coefficients with repeated observations: Part 1--Correlation within subjects. BMJ 310:446.

Bland JM, and Altman DG. 1995b. Calculating correlation coefficients with repeated observations: Part 2--Correlation between subjects. BMJ 310:633.

Blazevich AJ, Cannavan D, Coleman DR, and Horne S. 2007. Influence of concentric and eccentric resistance training on architectural adaptation in human quadriceps muscles. Journal of Applied Physiology 103:1565-1575. 10.1152/japplphysiol.00578.2007 
463 Blazevich AJ, Gill ND, and Zhou S. 2006. Intra- and intermuscular variation in human quadriceps

464

465

466

467

468

469

470

471

472

473

474

475

476

477

478

479

480

481

482

483

484

485

486

487

488

489

490

491

492

493

494

495

496

497

498

499

500

501

502

503

504

505

506 femoris architecture assessed in vivo. Journal of Anatomy 209:289-310. 10.1111/j.14697580.2006.00619.x

Borenstein M, Hedges LV, Higgins JPT, and Rothstein HR. 2009. Introduction to meta-analysis. Chichester, U.K.: John Wiley \& Sons.

Buckner SL, Dankel SJ, Mattocks KT, Jessee MB, Mouser JG, Counts BR, and Loenneke JP. 2016 a. The problem of muscle hypertrophy: Revisited. Muscle and Nerve 54:1012-1014. 10.1002 /mus. 25420

Buckner SL, Jessee MB, Mattocks KT, Mouser JG, Counts BR, Dankel SJ, and Loenneke JP. $2016 \mathrm{~b}$. Determining Strength: A Case for Multiple Methods of Measurement. Sports Medicine. 10.1007/s40279-016-0580-3

Churchward-Venne TA, Tieland M, Verdijk LB, Leenders M, Dirks ML, de Groot LC, and van Loon ᄂ. 2015. There Are No Nonresponders to Resistance-Type Exercise Training in Older Men and Women. Journal of the American Medical Directors Association 16:400-411. 10.1016/j.jamda.2015.01.071

Cooper HM, Hedges LV, and Valentine JC. 2009. The handbook of research synthesis and metaanalysis. New York: Russell Sage Foundation.

Corey DM, Dunlap WP, and Burke MJ. 1998. Averaging Correlations: Expected Values and Bias in Combined Pearson rs and Fisher's z Transformations. The Journal of General Psychology 125:245-261. 10.1080/00221309809595548

Cribb PJ, Williams AD, Stathis CG, Carey MF, and Hayes A. 2007. Effects of whey isolate, creatine, and resistance training on muscle hypertrophy. Medicine and Science in Sports and Exercise 39:298-307. 10.1249/01.mss.0000247002.32589.ef

Damas F, Phillips SM, Lixandrao ME, Vechin FC, Libardi CA, Roschel H, Tricoli V, and Ugrinowitsch C. 2016. Early resistance training-induced increases in muscle cross-sectional area are concomitant with edema-induced muscle swelling. European Journal of Applied Physiology 116:49-56. 10.1007/s00421-015-3243-4

Dankel SJ, Buckner SL, Jessee MB, Grant Mouser J, Mattocks KT, Abe T, and Loenneke JP. 2018. Correlations Do Not Show Cause and Effect: Not Even for Changes in Muscle Size and Strength. Sports Medicine 48:1-6. 10.1007/s40279-017-0774-3

DeLorme TL. 1945. Restoration of Muscle Power by Heavy-Resistance Exercises. JBJS 27:645-667.

Earp JE, Newton RU, Cormie P, and Blazevich AJ. 2015. Inhomogeneous Quadriceps Femoris Hypertrophy in Response to Strength and Power Training. Medicine and Science in Sports and Exercise 47:2389-2397. 10.1249/MSS.0000000000000669

Ema R, Wakahara T, Miyamoto N, Kanehisa H, and Kawakami Y. 2013. Inhomogeneous architectural changes of the quadriceps femoris induced by resistance training. European Journal of Applied Physiology 113:2691-2703. 10.1007/s00421-013-2700-1

Enoka RM. 1988. Muscle strength and its development. New perspectives. Sports Medicine 6:146-168.

Epp SS. 2011. Discrete mathematics with applications. Boston, MA: Brooks/Cole, Cengage Learning.

Erskine RM, Fletcher G, and Folland JP. 2014. The contribution of muscle hypertrophy to strength changes following resistance training. European Journal of Applied Physiology 114:12391249. 10.1007/s00421-014-2855-4 
507

508

509

510

511

512

513

514

515

516

517

518

519

520

521

522

523

524

525

526

527

528

529

530

531

532

533

534

535

536

537

538

539

540

541

542

543

544

545

546

547

548

549

Erskine RM, Jones DA, Williams AG, Stewart CE, and Degens H. 2010. Inter-individual variability in the adaptation of human muscle specific tension to progressive resistance training. European Journal of Applied Physiology 110:1117-1125. 10.1007/s00421-010-1601-9

Farina D. 2006. Interpretation of the surface electromyogram in dynamic contractions. Exercise and Sport Sciences Reviews 34:121-127.

Fluck M, and Hoppeler H. 2003. Molecular basis of skeletal muscle plasticity--from gene to form and function. Reviews of Physiology Biochemistry and Pharmacology 146:159-216. 10.1007/s10254-002-0004-7

Folland JP, and Williams AG. 2007. The adaptations to strength training : morphological and neurological contributions to increased strength. Sports Medicine 37:145-168.

Franchi MV, Longo S, Mallinson J, Quinlan JI, Taylor T, Greenhaff PL, and Narici MV. 2017. Muscle thickness correlates to muscle cross sectional area in the assessment of strength training induced hypertrophy. Scandinavian Journal of Medicine and Science in Sports:Epub ahead of print. 10.1111/sms.12961

Fukunaga T, Miyatani M, Tachi M, Kouzaki M, Kawakami Y, and Kanehisa H. 2001. Muscle volume is a major determinant of joint torque in humans. Acta Physiologica Scandinavica 172:249-255. 10.1046/j.1365-201x.2001.00867.x

Fukunaga T, Roy RR, Shellock FG, Hodgson JA, and Edgerton VR. 1996. Specific tension of human plantar flexors and dorsiflexors. Journal of Applied Physiology 80:158-165.

Fuller WA. 1987. Measurement error models. New York: Wiley.

Gelman A, and Hill J. 2007. Data analysis using regression and multilevel/hierarchical models. Cambridge ; New York: Cambridge University Press.

Goldstein H. 2011. Multilevel statistical models. Chichester, West Sussex: Wiley.

Haberfehlner H, Maas H, Harlaar J, Becher JG, Buizer Al, and Jaspers RT. 2016. Freehand threedimensional ultrasound to assess semitendinosus muscle morphology. Journal of Anatomy 229:591-599. 10.1111/joa.12501

Hall W. 1987. A simplified logic of causal inference. Australian and New Zealand Journal of Psychiatry 21:507-513. 10.3109/00048678709158918

Hedges LV, and Olkin I. 1985. Statistical methods for meta-analysis. Orlando: Academic Press. Hopkins WG. 2002. A scale of magnitudes for effect statistics. A new view of statistics 502.

Jackson C, Best N, and Richardson S. 2006. Improving ecological inference using individual-level data. Statistics in Medicine 25:2136-2159. 10.1002/sim.2370

Jenkins ND, Miller JM, Buckner SL, Cochrane KC, Bergstrom HC, Hill EC, Smith CM, Housh TJ, and Cramer JT. 2015. Test-Retest Reliability of Single Transverse versus Panoramic Ultrasound Imaging for Muscle Size and Echo Intensity of the Biceps Brachii. Ultrasound in Medicine and Biology 41:1584-1591. 10.1016/j.ultrasmedbio.2015.01.017

Jenkins NDM, Miramonti AA, Hill EC, Smith CM, Cochrane-Snyman KC, Housh TJ, and Cramer JT. 2017. Greater Neural Adaptations following High- vs. Low-Load Resistance Training. Frontiers in Physiology 8:331. 10.3389/fphys.2017.00331

Jones DA, Rutherford OM, and Parker DF. 1989. Physiological changes in skeletal muscle as a result of strength training. Quarterly Journal of Experimental Physiology 74:233-256.

Karelis AD, Chamberland G, Aubertin-Leheudre M, Duval C, Ecological mobility in A, and Parkinson g. 2013. Validation of a portable bioelectrical impedance analyzer for the 
550

551

552

553

554

555

556

557

558

559

560

561

562

563

564

565

566

567

568

569

570

571

572

573

574

575

576

577

578

579

580

581

582

583

584

585

586

587

588

589

590

591

592

assessment of body composition. Applied Physiology, Nutrition, and Metabolism Physiologie Appliquée, Nutrition et Métabolisme 38:27-32. 10.1139/apnm-2012-0129

Koo TK, and Li MY. 2016. A Guideline of Selecting and Reporting Intraclass Correlation Coefficients for Reliability Research. Journal of Chiropractic Medicine 15:155-163. 10.1016/j.jcm.2016.02.012

Lieber RL, and Ward SR. 2011. Skeletal muscle design to meet functional demands. Philosophical Transactions of the Royal Society of London Series B: Biological Sciences 366:1466-1476. 10.1098/rstb.2010.0316

Loenneke JP, Rossow LM, Fahs CA, Thiebaud RS, Grant Mouser J, and Bemben MG. 2017. Timecourse of muscle growth, and its relationship with muscle strength in both young and older women. Geriatr Gerontol Int. 10.1111/ggi.13010

Maeo S, Shan X, Otsuka S, Kanehisa H, and Kawakami Y. 2018. Neuromuscular Adaptations to Work-matched Maximal Eccentric vs Concentric Training. Medicine and Science in Sports and Exercise. 10.1249/MSS.0000000000001611

Mattocks KT, Buckner SL, Jessee MB, Dankel SJ, Mouser JG, and Loenneke JP. 2017. Practicing the Test Produces Strength Equivalent To Higher Volume Training. Medicine and Science in Sports and Exercise. 10.1249/MSS.0000000000001300

Maughan RJ, and Nimmo MA. 1984. The influence of variations in muscle fibre composition on muscle strength and cross-sectional area in untrained males. Journal of Physiology 351:299-311.

Maughan RJ, Watson JS, and Weir J. 1984. Muscle strength and cross-sectional area in man: a comparison of strength-trained and untrained subjects. British Journal of Sports Medicine 18:149-157.

Narici MV, Hoppeler H, Kayser B, Landoni L, Claassen H, Gavardi C, Conti M, and Cerretelli P. 1996. Human quadriceps cross-sectional area, torque and neural activation during 6 months strength training. Acta Physiologica Scandinavica 157:175-186. 10.1046/j.1365201X.1996.483230000.x

Noorkoiv M, Nosaka K, and Blazevich AJ. 2014. Neuromuscular adaptations associated with knee joint angle-specific force change. Medicine and Science in Sports and Exercise 46:15251537. 10.1249/MSS.0000000000000269

Pope ZK, Hester GM, Benik FM, and DeFreitas JM. 2016. Action potential amplitude as a noninvasive indicator of motor unit-specific hypertrophy. Journal of Neurophysiology 115:2608-2614. 10.1152/jn.00039.2016

Powell PL, Roy RR, Kanim P, Bello MA, and Edgerton VR. 1984. Predictability of skeletal muscle tension from architectural determinations in guinea pig hindlimbs. J Appl Physiol Respir Environ Exerc Physiol 57:1715-1721.

Quené $\mathrm{H}$, and van den Bergh H. 2004. On multi-level modeling of data from repeated measures designs: a tutorial. Speech Communication 43:103-121. http://dx.doi.org/10.1016/i.specom.2004.02.004

R Core Development Team. 2017. R: A language and environment for statistical computing. Austria, Vienna: R Foundation for Statistical Computing.

Ralston GW, Kilgore L, Wyatt FB, and Baker JS. 2017. The Effect of Weekly Set Volume on Strength Gain: A Meta-Analysis. Sports Medicine. 10.1007/s40279-017-0762-7 
593

594

595

596

597

598

599

600

601

602

603

604

605

606

607

608

609

610

611

612

613

614

615

616

617

618

619

620

621

622

623

624

625

626

627

628

629

630

631

632

633

634

Rasch PJ, and Morehouse LE. 1957. Effect of static and dynamic exercises on muscular strength and hypertrophy. Journal of Applied Physiology 11:29-34. 10.1152/jappl.1957.11.1.29

Raudenbush SW, and Bryk AS. 2002. Hierarchical linear models : applications and data analysis methods. Thousand Oaks: Sage Publications.

Riegelman R. 1979. Contributory cause: unnecessary and insufficient. Postgraduate Medicine 66:177-179.

Robinson WS. 1950. Ecological Correlations and the Behavior of Individuals. American Sociological Review 15:351-357. 10.2307/2087176

Schantz P, Randall-Fox E, Hutchison W, Tyden A, and Astrand PO. 1983. Muscle fibre type distribution, muscle cross-sectional area and maximal voluntary strength in humans. Acta Physiologica Scandinavica 117:219-226. 10.1111/j.1748-1716.1983.tb07200.x

Schoenfeld BJ, Ogborn D, and Krieger JW. 2017. Dose-response relationship between weekly resistance training volume and increases in muscle mass: A systematic review and metaanalysis. Journal of Sports Sciences 35:1073-1082. 10.1080/02640414.2016.1210197

Sugisaki N, Wakahara T, Murata K, Miyamoto N, Kawakami Y, Kanehisa H, and Fukunaga T. 2015. Influence of muscle hypertrophy on the moment arm of the triceps brachii muscle. Journal of Applied Biomechanics 31:111-116. 10.1123/jab.2014-0126

Than C, Tosovic D, Seidl L, and Mark Brown J. 2016. The effect of exercise hypertrophy and disuse atrophy on muscle contractile properties: a mechanomyographic analysis. European Journal of Applied Physiology 116:2155-2165. 10.1007/s00421-016-3469-9

Trezise J, Collier N, and Blazevich AJ. 2016. Anatomical and neuromuscular variables strongly predict maximum knee extension torque in healthy men. European Journal of Applied Physiology 116:1159-1177. 10.1007/s00421-016-3352-8

Vigotsky AD, Bryanton MA, Nuckols G, Beardsley C, Contreras B, Evans J, and Schoenfeld BJ. 2018. Biomechanical, anthropometric, and psychological determinants of barbell back squat strength. Journal of Strength and Conditioning Research. 10.1519/JSC.00000000000002535

Vigotsky AD, Contreras B, and Beardsley C. 2015. Biomechanical implications of skeletal muscle hypertrophy and atrophy: a musculoskeletal model. PeerJ 3:e1462. 10.7717/peerj.1462

Vigotsky AD, Halperin I, Lehman GJ, Trajano GS, and Vieira TM. 2017. Interpreting Signal Amplitudes in Surface Electromyography Studies in Sport and Rehabilitation Sciences. Frontiers in Physiology 8:985. 10.3389/fphys.2017.00985

Wakahara T, Fukutani A, Kawakami Y, and Yanai T. 2013. Nonuniform muscle hypertrophy: its relation to muscle activation in training session. Medicine and Science in Sports and Exercise 45:2158-2165. 10.1249/MSS.0b013e3182995349

Wakahara T, Miyamoto N, Sugisaki N, Murata K, Kanehisa H, Kawakami Y, Fukunaga T, and Yanai T. 2012. Association between regional differences in muscle activation in one session of resistance exercise and in muscle hypertrophy after resistance training. European Journal of Applied Physiology 112:1569-1576. 10.1007/s00421-011-2121-y

Ward SR, Eng CM, Smallwood LH, and Lieber RL. 2009. Are current measurements of lower extremity muscle architecture accurate? Clinical Orthopaedics and Related Research 467:1074-1082. 10.1007/s11999-008-0594-8 
635 Watanabe K, Kouzaki M, Ogawa M, Akima H, and Moritani T. 2018. Relationships between muscle 636 strength and multi-channel surface EMG parameters in eighty-eight elderly. European 637 Review of Aging and Physical Activity 15:3. 10.1186/s11556-018-0192-z

638 


\section{Table $\mathbf{1}$ (on next page)}

Correlation coefficient and variance accounted for interpretations.

Adapted from Hopkins (2002). Note that all intervals are of the form $x_{\text {low }} \leq x_{0}<x_{\text {high }}$. 
1 Table 1. Correlation coefficient and variance accounted for interpretations.

\begin{tabular}{|l|l|l|}
\hline Interpretation & Correlation coefficient $(\boldsymbol{r}$ or $\boldsymbol{\rho})$ & Variance accounted for (\%) \\
\hline Trivial & {$[0,0.1)$} & {$[0,1)$} \\
\hline Small & {$[0.1,0.3)$} & {$[1,9)$} \\
\hline Moderate & {$[0.3,0.5)$} & {$[9,25)$} \\
\hline Large/strong & {$[0.5,0.7)$} & {$[25,49)$} \\
\hline Very large/strong & {$[0.7,0.9)$} & {$[49,81)$} \\
\hline Nearly perfect & {$[0.9,1)$} & {$[81,100)$} \\
\hline Perfect & 1 & 100 \\
\hline
\end{tabular}

2 Adapted from Hopkins (2002). Note that all intervals are of the form $x_{\text {low }} \leq x_{o}<x_{\text {high }}$. 


\section{Table 2 (on next page)}

Percent (\%) variance accounted for $(95 \% \mathrm{Cl})$ using different types of models.

$30 \%, 50 \%$, and $70 \%$ represent the position of the ultrasound probe on the brachium. Average represents the average of all three of the measured thicknesses at a given time point. Crosssectional area was measured at 50\%. ANCOVA $=$ analysis of covariance; $\mathrm{HLM}=$ hierarchical linear model 
1 Table 2. Percent (\%) variance accounted for (95\% CI) using different types of models.

\begin{tabular}{|l|l|l|l|l|}
\hline \multirow{2}{*}{ Measure } & \multicolumn{3}{|l|}{ Between-subjects } & Within-subjects \\
\cline { 2 - 5 } & Training & Detraining & ANCOVA & HLM \\
\hline Thickness (30\%) & $3.6(0-61.9)$ & $1.0(0-45.1)$ & $0.2(0-6.1)$ & $7.4(0.8-16.0)$ \\
\hline Thickness (50\%) & $0.8(0-21.6)$ & $0.0(0-23.7)$ & $0.3(0-9.7)$ & $24.1(6.7-42.0)$ \\
\hline Thickness (70\%) & $1.4(0-39.1)$ & $1.6(0-38.0)$ & $2.2(0-10.9)$ & $7.5(2.1-23.7)$ \\
\hline Thickness (Average) & $0.4(0-21.1)$ & $0.0(0-26.4)$ & $1.2(0-12.9)$ & $18.1(6.6-30.4)$ \\
\hline Cross-sectional area & $0.4(0-32.2)$ & $1.2(0-35.4)$ & $11.7(1.1-34.2)$ & $12.1(2.0-69.5)$ \\
\hline
\end{tabular}

$230 \%, 50 \%$, and $70 \%$ represent the position of the ultrasound probe on the brachium. Average represents the average

3 of all three of the measured thicknesses at a given time point. Cross-sectional area was measured at $50 \%$. ANCOVA

4 = analysis of covariance; HLM = hierarchical linear model 


\section{Table 3(on next page)}

Correlation matrix of measures of muscle size.

$30 \%, 50 \%$, and $70 \%$ represent the position of the ultrasound probe on the brachium. Average represents the average of all three of the measured thicknesses at a given time point. Crosssectional area was measured at $50 \%{ }^{*}=$ between-subject correlation; $\dagger=$ weighted withinsubject correlation 
1 Table 3. Correlation matrix of measures of muscle size.

\begin{tabular}{|l|l|l|l|l|l|}
\hline & $\begin{array}{l}\text { Thickness } \\
(30 \%)\end{array}$ & $\begin{array}{l}\text { Thickness } \\
(50 \%)\end{array}$ & $\begin{array}{l}\text { Thickness } \\
(70 \%)\end{array}$ & $\begin{array}{l}\text { Thickness } \\
\text { (Average) }\end{array}$ & $\begin{array}{l}\text { Cross-sectional } \\
\text { area }\end{array}$ \\
\hline $\begin{array}{l}\text { Thickness } \\
(30 \%)\end{array}$ & $0.503^{*}$ & $0.618^{*}$ & $0.778^{*}$ & $0.557^{*}$ \\
\hline $\begin{array}{l}\text { Thickness } \\
(50 \%)\end{array}$ & $0.344^{\dagger}$ & $0.687^{\dagger}$ & $0.869^{*}$ & $0.916^{*}$ & $0.742^{*}$ \\
\hline $\begin{array}{l}\text { Thickness } \\
(70 \%)\end{array}$ & $0.326^{\dagger}$ & $0.875^{\dagger}$ & $0.871^{\dagger}$ & $0.945^{*}$ & $0.730^{*}$ \\
\hline $\begin{array}{l}\text { Thickness } \\
(\text { Average })\end{array}$ & $0.659^{\dagger}$ & & & & $0.773 *$ \\
\hline $\begin{array}{l}\text { Cross- } \\
\text { sectional area }\end{array}$ & & & & & \\
\hline
\end{tabular}

$230 \%, 50 \%$, and $70 \%$ represent the position of the ultrasound probe on the brachium. Average represents the average 3 of all three of the measured thicknesses at a given time point. Cross-sectional area was measured at $50 \%$. $*=$ between-

4 subject correlation; $\dagger=$ weighted within-subject correlation 